\title{
Deduction of the conformation of short chain luminescent conjugated polymers from single molecule photophysics
}

\author{
J.D. White ${ }^{\mathrm{a}, \mathrm{b}}$, J.H. Hsu ${ }^{\text {c }}$, W.S. Fann ${ }^{\mathrm{a}, \mathrm{d}, *}$, Shu-Chun Yang ${ }^{\mathrm{a}}$, G.Y. Pern ${ }^{\mathrm{e}}$, \\ S.A. Chen ${ }^{\mathrm{e}}$ \\ a Institute of Atomic and Molecular Sciences, Academica Sinica, P.O. Box 23-166, Taipei, Taiwan, ROC \\ ${ }^{\mathrm{b}}$ Centre for Highspeed and Broadband Networking, Multimedia University, Malaysia \\ ${ }^{\mathrm{c}}$ Department of Chemistry, National Taiwan University, Taipei, Taiwan, ROC \\ ${ }^{\mathrm{d}}$ Department of Physics, National Taiwan University, Taipei, Taiwan, ROC \\ e Department of Chemical Engineering, National Tsing-Hwa University, Hsinchu, Taiwan, ROC
}

Received 10 January 2001; in final form 12 March 2001

\begin{abstract}
The fluorescent transient of single polymers of a PPV derivative, poly(2,5-dioctyloxy $p$-phenylene vinylene) (DOOPPV) spin-cast in polystyrene matrix is observed. Having the polymer with low-defect density and length close to its persistent length, the effect of defects on conformation can be investigated. Independent chromophores align in the majority of cases suggesting that the polymer maintains a rod-like structure in the absence of defects. Non-aligned chromophores are the result of defects that introduce 'kinks' in the polymer conformation. (c) 2001 Published by Elsevier Science B.V.
\end{abstract}

Single molecule detection has been proven to be an excellent method for studying the photophysics of molecules without blurring due to ensemble averaging. Since the first observation of a single dye molecule at room temperature in 1993 [1], many groups have undertaken studies of similar small molecules showing that they behave as a single quantum system [2-6]. Nimitz et al.'s (1996) discovery that a nanocrystal - although composed of many CdSe molecules [7] - also behaves as a single quantum system led to many studies seeking to understand the phenomena [8]. While some groups have concentrated on the small molecules, others

\footnotetext{
${ }^{*}$ Corresonding author. Fax: +886-2-2362-0200.

E-mail address: fann@gate.sinica.edu.tw (W.S. Fann).
}

have sought to observe similar phenomena in larger molecular systems $[6,9,10]$. Special attention has been paid to the light emitting conjugated polymer thin films [11-16] due to their potential as the active medium in OLED devices [17]. The results reported for these large systems - composed of thousands of monomers have been, to say the least, confusing. This is not only due to the size of the system and its many potential conformations [15] but also due to complications resulting from the random spacing of intrinsic defects [13] - typically on the order of 5\%. For example, MEH-PPV polymer commonly studied contains over 1700 vinyl bonds of which some 100 are statistically defective. Hu et al. have recently shown that these defects result in conformations quite different than that expected from a perfect system [13]. 
In this Letter we report on the study of an intermediate-sized polymer - larger than the small molecules previously studied and much shorter than the polymer chains commonly being investigated. By studying a polymer having a length close to the persistence length for this polymer in solution [18] we are able to reduce the number of possible conformations. Indeed, the polymer of this length is expected to be predominately rodshaped. In addition, by preparing this polymer with a defect rate of $\sim 1 \%$ (defect rate $<1.5 \%$ as measured by NMR using the technique of Becker [19], limited by $\mathrm{S} / \mathrm{N}$ ratio), $75 \%$ of the polymers will be defect free and only 5\% will contain more than a single defect. Thus the photophysics of both the defect-free polymer and the effect of a single defect can be conveniently studied. Indeed we believe that a thorough study of this sort of intermediate-sized system provides great possibility for the quantitative understanding of the behaviors in conjugated polymers.

In the work described here, pristine poly $(2,5-$ dioctyloxy $p$-phenylene vinylene) (DOO-PPV) [20] was fractionalized by taking advantage of the limited solubility of this symmetrically substituted structure [21]. The solid polymer was first immersed in chloroform at $0^{\circ} \mathrm{C}$ for a few hours. The dissolved polymer (short chain lengths) was then discarded while the residual polymer was dried and dissolved in chloroform at $24^{\circ} \mathrm{C}$. In this step the residual polymer (long chain lengths) is discarded. The GPC measurements shows that the dissolved polymers have a number average molecular weight $\left(M_{\mathrm{n}}\right)$ of $8.2 \mathrm{kDa}$ and a polydispersity, $M_{\mathrm{w}} / M_{\mathrm{n}}$, of 2.23 , where $M_{\mathrm{w}}$ is the weight average molecular weight. Since in single molecule experiments the molecule is measured one at a time, it is more appropriate to use $M_{\mathrm{n}}$ than the standard 'meanaverage' molecular weight $\left(M_{\mathrm{n}} M_{\mathrm{w}}\right)^{1 / 2}$. The $M_{\mathrm{n}}$ corresponds to polymer of about 24 monomers. Assuming that the 1D excitons are delocalized along the backbone over approximately 6 monomers, this length corresponds to a polymer of $\sim 4$ chromophores and $13 \mathrm{~nm}$ in length (close to the persistence length for this type of polymer) $[18,22]$.

After dilution of the polymer in an inert polystyrene matrix, it was spin-cast onto a fused silica substrate. The resulting film thickness was $<100$ nm. Under these conditions, most molecules will align to have the polymer backbone in the plane of the thin film. As oxygen and water have been shown to be extremely detrimental to polymer lifetime [14,16], extensive steps were taken to protect the polymer from exposure to oxygen and water. $\mathrm{N}_{2}$ was bubbled through all solutions, drying after spin-casting was done in a low-pressure nitrogen environment and a special holder designed to ensure that the sample surface remained in contact with $\mathrm{N}_{2}$ only during imaging (Fig. 1 inset).

The sample was observed using confocal fluorescence microscopy (Fig. 1). Three criteria were used to ascertain that we were, in fact, looking at single polymers and not aggregates. An area scan was used to check that the bright spots were diffraction limited. Sample concentration was varied over two order of magnitude (from 200 to 2 molecules $/ 1000 \mu \mathrm{m}^{2}$ - data being recorded taken at the lower concentrations) and the density of the spots seen to vary appropriately. Finally, the ensemble average of individual molecule spectrum was taken and found to be similar to that of the dilute polymer measured in solution [16,20].

Having established that one is looking at single polymers, the first questions to be answered are whether or not there is any alignment of chromophore absorption dipoles within a single polymer (i.e., are all dipoles in the same direction), and whether or not there is a preferential orientation for an ensemble of polymers resulting from the spin-coating process. Comparing the fluorescence intensity of two successive area scans, one taken with linearly polarized light and one in which the direction of the electric field vector is modulated rapidly (compared to integration time) at constant angular frequency, answers both questions. Since the intensity of the emission signal $I(t)$ is proportional to the absorption, which according the dipole approximation, is proportional to $\left\langle(\boldsymbol{\mu} \cdot \boldsymbol{E})^{2}\right\rangle$, where $\boldsymbol{\mu}$ is the molecular transition dipole and $\boldsymbol{E}(t)$ is the laser field. Thus by modulating the laser field one averages over all possible dipole directions while maintaining the polarization direction selectively excites those molecules whose dipoles are aligned with the electric field. For polymers having approximately the same length, one expects that 


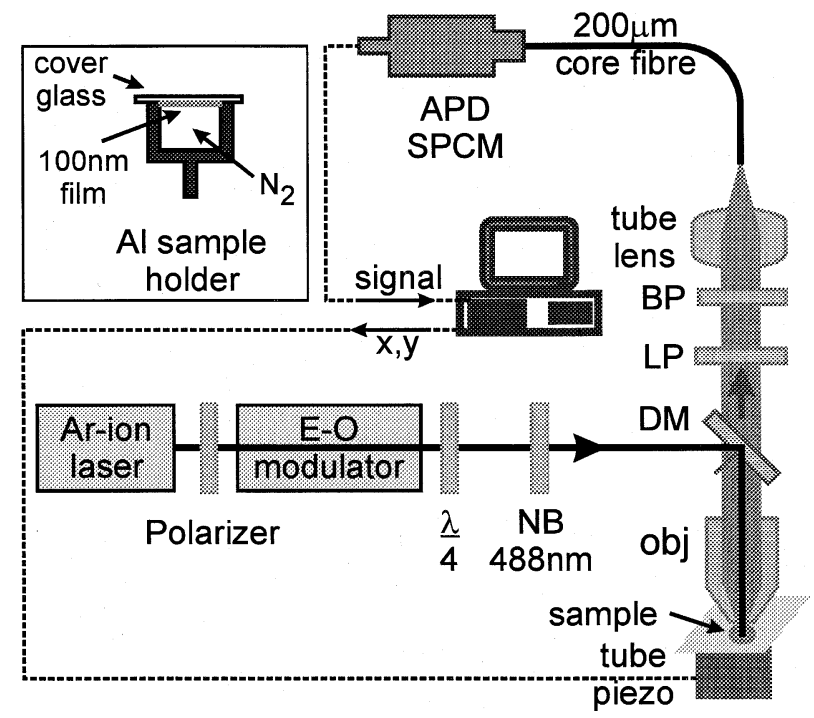

Fig. 1. Schematic of the single molecule confocal microscope. In all cases continuous TEMoo linearly polarized laser light with a near diffraction limited spot ( $300 \mathrm{~nm}$ ) after passing through a $488 \mathrm{~nm}$ narrow band (NB) filter and dichroic mirror (DM) through an oil immersion $100 \times$ objective lens $(\mathrm{NA}=1.3)$ was used to excite the sample. Fluorescence was collected in the common epifluorescence geometry. After passage through a longpass (LP) and $70 \mathrm{~nm}$ bandpass (BP) filter centered at $550 \mathrm{~nm}$ to eliminate scattered $488 \mathrm{~nm}$ light, it was focused onto a $\varnothing 200 \mu \mathrm{m}$ core optical fiber for detection with an APD operating in photon counting mode with an integration time $10 \mathrm{~ms}$. Area scans were accomplished by moving the sample with a tube peizoelectric. Fluorescence transients were recorded after centering the light on individual polymers. The E-O modulator in the excitation path allows the electric field vector to be rotated either slowly or rapidly with respect to the integration time [see text for details]. The inset shows the sample holder used to place the sample in an $\mathrm{N}_{2}$ environment during scanning.

the modulated fluorescence intensity will be approximately the same for all polymers, while the peak intensity of polymers will vary in the linearly polarized case from 0 to 2 times the modulated case if there is an alignment of dipoles within individual polymers. Such a variation with some molecules increasing in brightness while others fade into the background was observed. We conclude the correlative orientation of chromophores within an individual polymer.

Further light is shed on the relative direction of individual chromophores by investigating the fluorescent transients of individual polymers taken with unmodulated cw linearly polarized light. Two different types of behavior are possible. On the one hand, if the polymer has maintained a rigid rod structure when spin-cast then one expects that the chromophore dipoles will align predominately in the same direction and the fluorescent transient will exhibit a decay structure dominated by multiple equal steps. On the other hand, if the struc- ture is not linear and the dipoles do not align then one would still expect to see steps; however, in this case the magnitude of the steps may be unequal. The transient trace shown in Fig. 2 is typical of that observed for the vast majority of the single polymers studied $(\sim 100)$. Fluorescence is seen to occur at three discrete intensity levels equally separated by 6 counts $/ 10 \mathrm{~ms}$ (background is 2 counts/ $10 \mathrm{~ms}$ ) - the final discrete step back to background is not shown in this figure so as to emphasis the first part of the trace. This result can be interpreted as follows. Initially three semi-independent aligned chromophores (within a single polymer) are active. After $330 \mathrm{~ms}$, one chromophore enters a temporary 'dark state' while the other two remain active. $13.5 \mathrm{~s}$ into the trace this chromophore abruptly leaves the dark state and resumes emitting. At the $20 \mathrm{~s}$ point another (or the same) chromophore enters a temporary dark state until the $23 \mathrm{~s}$ point. All three chomophores continue to be active for $30 \mathrm{~s}$, whereupon 2 chromophores are permanently 


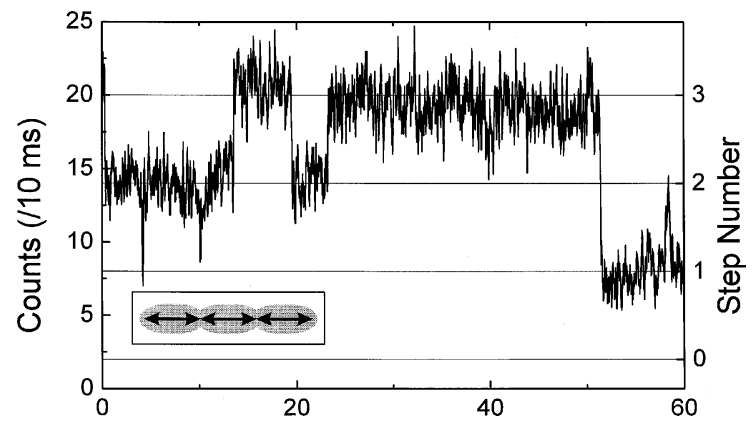

Fig. 2. Typical fluorescent transient of a single DOO-PPV molecule that is presumed to be defect free [see text]. Excitation irradiance is $200 \mathrm{~W} / \mathrm{cm}^{2}$. Background is $\sim 2$ counts $/ 10 \mathrm{~ms}$. Note that only the first $60 \mathrm{~s}$ of this scan are shown in order so that the individual steps are not obscured. Note that the step sizes are all of approximately the same height. The inset shows the alignment of chromophores relative to electric field that is expected to generate this type of fluorescent transient. Due to the lowexcitation irradiance, signal to noise ratio has been increased by continuous averaging of 10 adjacent bins (this figure only). The inset shows the deduced conformation of this polymer.

quenched while the final chromophore continues to emit until the end of the recorded trace. In the majority of cases the model of 3 or 4 aligned chromophores successfully explains the observed data indicating that the spin-cast polymer maintains a rigid rod-like structure.

However, although this represents the majority of the molecules observed, a significant minority, while exhibiting emission at multiple discrete levels, does not have steps of equal magnitude. The fluorescent transient of a polymer exhibiting this type of behavior is shown in Fig. 3. Note that in this figure the excitation irradiance has been increased by a factor of eight so that the smaller steps can be clearly observed. This polymer exhibits emission at three discrete levels, first at around 48 counts, followed by 11 and finally 5 counts above background per 10 millisecond time interval. This data can be understood as follows. For the first $36 \mathrm{~s}$ three chromophores are active one chromophore is closely aligned with the $\boldsymbol{E}(t)$ while the remaining two are at an angle. At $41 \mathrm{~s}$, the chromophore whose dipole was most closely aligned with the excitation field (and hence has absorbed the most photons) is abruptly quenched. The remaining two chromophores (aligned) re-

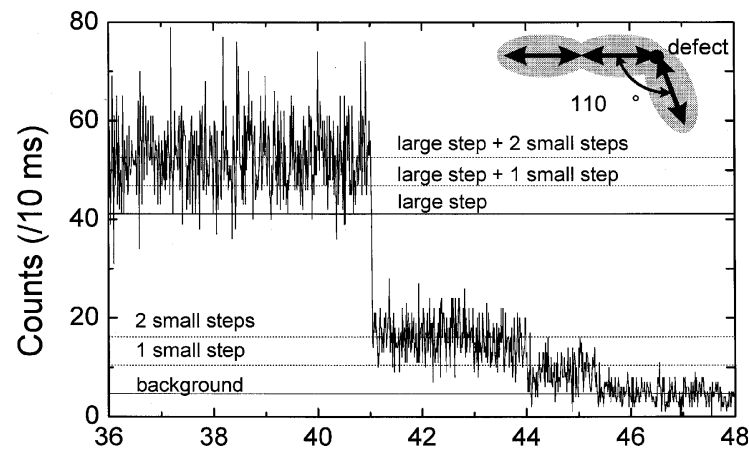

Fig. 3. Typical fluorescent transient of a single DOO-PPV polymer that is believed to contain a single defect [see text]. High excitation irradiance was used $\left(1600 \mathrm{~W} / \mathrm{cm}^{2}\right)$ to clearly show the non-constant step height. Background is $\sim 5$ counts $/ 10$ $\mathrm{ms}$. The non-constant step size indicates that the dipoles are not all aligned to the same direction. The inset shows one of the two possible conformations that fit the above data.

main active being independently quenched at 44 and $45.3 \mathrm{~s}$. From the ratio of the size of the large and small steps (7.17) and assuming that the angle between the strongest chromophore's $\boldsymbol{\mu}$ and $\boldsymbol{E}(t)$ is $<35^{\circ}$, the angle between chromophores can be calculated to be either $110 \pm 2^{\circ}$ or $70 \pm 2^{\circ}$. The polymer is thus no longer straight but contains a single 'kink'. The inset to the figure shows one of the two possible configurations $\left(110 \pm 2^{\circ}\right)$. Recently $\mathrm{Hu}$ et al. [13], have discussed such an effect from defects by means of Monte Carlo simulation showing the role defects play in determining the conformation of large polymers showing that a single defect can create just such a kink.

This interpretation can be checked by recording the fluorescent transient as $\boldsymbol{E}(t)$ is modulated slowly relative to the integration time and quickly relative to fluorescence decay [23]. In this case $\boldsymbol{E}(t)$ was modulated at $2.5 \mathrm{~Hz}$. By least squares fitting each section of the curve by $I=(\boldsymbol{\mu} \cdot \boldsymbol{E})^{2}=$ $A \sin ^{2}(\omega t+\varphi)+C$, (where $I$ is the fluorescence intensity, $\omega$ is the modulation frequency and $A, \varphi$, $C$ are allowed to vary) and assuming that each chromophore emits photons at the same rate, one can deduce the relative orientations of the chromophore dipoles. For aligned polymers, the maximum intensity of aligned dipoles was an integral multiple of a base intensity, modulation was $100 \%$, and $\varphi$ remained constant. Fig. 4 shows the results 


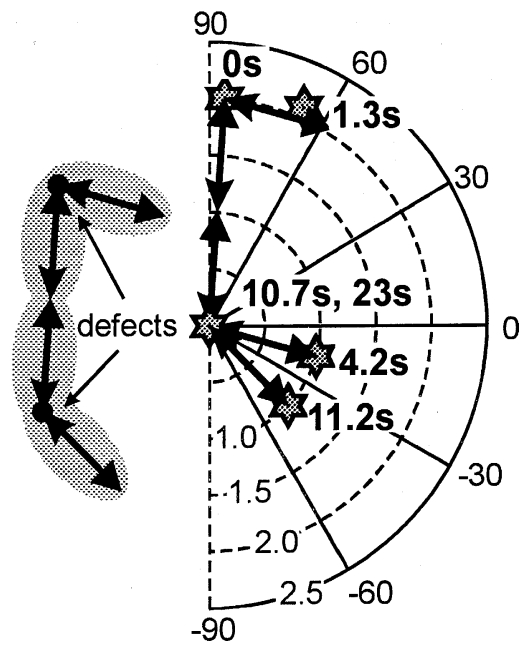

Fig. 4. Comparison of the results of curve fitting (to $\left.I=A \sin ^{2}(\omega t+\varphi)+C\right)$ the modulated polarization fluorescent transient of a polymer with a model of the polymer as four chromophores and two defects [see text]. In this polar graph, the values of $A+C$ vs. $\varphi$ as the molecule decays are plotted as 6-pointed stars and labeled with the time at which the transition to this emission state occurs. The amplitudes, $1,1.5$ and 2 are denoted in terms of the fundamental step size observed. Superimposed using arrows are the combination of dipoles that account for observed emission at each time. The distance between the origin and the corresponding time represents the observed emission intensity. The inset shows one possible polymer structure that can account for this behavior - a combination of four chromophores and two defects.

of such a procedure (for the more interesting case of a relatively rare 'defect-rich' polymer), with the fitted values represented by stars. A simple model representing the polymer as four chromophores and two defects inspired kinks is seen to closely model the observed data. During the initial seconds two parallel chromophores (1 and 2$)$ are active. These two chromophores are soon (at $1.3 \mathrm{~s}$ ) joined by a third chromophore (3) (separated from the first 2 by a defect). At $4.2 \mathrm{~s}$ two chromophores (1 and 2) are permanently quenched, while chromophore 3 continues to be active until the $10.7 \mathrm{~s}$ point. For the next half second there is no emission until chromophore 4 becomes active, continuing to emit until $23 \mathrm{~s}$ when it also is permanently quenched. One possible configuration for this polymer, incorporating two defects is shown on the left side of Fig. 4.
Summarizing, well-defined steps indicate the existence of well defined absorption chromophores in polymers with length close the persistence length (likely result of their low molecular weight and hence limited number of conformations). The conformation of such polymers can be deduced from their photophysical properties. For the majority of polymers, the fluorescent transient decay exhibits equal intensity steps indicating an alignment of chromophore dipoles implying that the pristine defect polymer has taken a rodshaped structure. Unequal steps imply a misalignment of dipoles caused by kinks in the otherwise straight polymer. These kinks are most likely caused by the existence of defects. Thus by observing the fluorescent transient decay of a single polymer not only its conformation but also the existence and number of defects in its backbone can be deduced.

\section{Acknowledgements}

This research is supported by the National Science Council, ROC. We gratefully acknowledge helpful comments by J. Yu and the reviewer, as well as the help of T.Y. Luh's laboratory for the NMR measurements.

\section{References}

[1] E. Betzig, R.J. Chichester, Science 262 (1993) 1422.

[2] X.S. Xie, R.C. Dunn, Science 265 (1994) 361.

[3] W.P. Ambrose, P.M. Goodwin, J.C. Martin, R.A. Keller, Science 265 (1994) 364.

[4] S. Nie, S.R. Emory, Science 275 (1997) 1102.

[5] X.H. Xu, E.S. Yeung, Science 275 (1997) 1106.

[6] W.E. Moerner, Science 277 (1997) 1059.

[7] M. Nirmal, B.O. Dabbousi, M.G. Bawendi, J.J. Macklin, J.K. Trautman, T.D. Harris, L.E. Brus, Nature 383 (1996) 802.

[8] Julia Uppenbrink, Daniel Clery (Eds.), special issue reviewing single molecules Science 283 (1999) 1668.

[9] L. Ying, X.S. Xie, J. Phys. Chem. B 102 (1998) 10399.

[10] M.A. Bopp, A. Sytnik, T.D. Howard, R.J. Cogdell, R.M. Hochstrasser, Proc. Natl. Acad. Sci. USA 96 (1999) 11271.

[11] D.A. Vanden Bout, W.T. Yip, D. Hu, D.K. Fu, T.M. Swager, P.F. Barbara, Science 277 (1997) 1074. 
[12] D. Hu, J. Yu, P.F. Barbara, J. Am. Chem. Soc. 121 (1999) 6936.

[13] D. Hu, J. Yu, K. Wong, B. Bagchi, P.J. Rossky, P.F. Barbara, Nature 405 (2000) 1030.

[14] J. Yu, D. Hu, P.F. Barbara, Science 289 (2000) 1327.

[15] T. Huser, M. Yan, L.J. Rothberg, Proc. Natl. Acad. Sci. USA 97 (2000) 11187.

[16] J.D. White, J.H. Hsu, S.C. Yang, W.S. Fann, G.Y. Pern, S.A. Chen, J. Chem. Phys. 114 (2001) 3848.

[17] R.H. Friend, R.W. Gymer, A.B. Holmes, J.H. Burroughes, R.N. Marks, C. Taliani, D.D.C. Bradley, D.A. Dos Santos, J.L. Brédas, M. Lögdlund, W.R. Salaneck, Nature 397 (1999) 121
[18] C.L. Gettinger, A.J. Heeger, J.M. Drake, D.J. Pine, J. Chem. Phys. 101 (1994) 1673.

[19] H. Becker, H. Spreitzer, K. Ibrom, W. Kreuder, Macromolecules 32 (1999) 4925.

[20] J.H. Hsu, W. Fann, P.H. Tsao, K.R. Chuang, S.A. Chen, J. Phys. Chem. A 103 (1999) 2375.

[21] J.H. Hsu, M. Hayashi, S.H. Lin, W. Fann, L.J. Rothberg, K.R. Chuang, S.A. Chen, unpublished.

[22] M. Yan, L.J. Rothberg, F. Papadimitrakopoulos, M.E. Galvin, T.M. Miller, Phys. Rev. Lett. 73 (1994) 744.

[23] T. Ha, J. Glass, Th. Enderle, D.S. Chemla, S. Weiss, Phys. Rev. Lett. 80 (1998) 2093. 